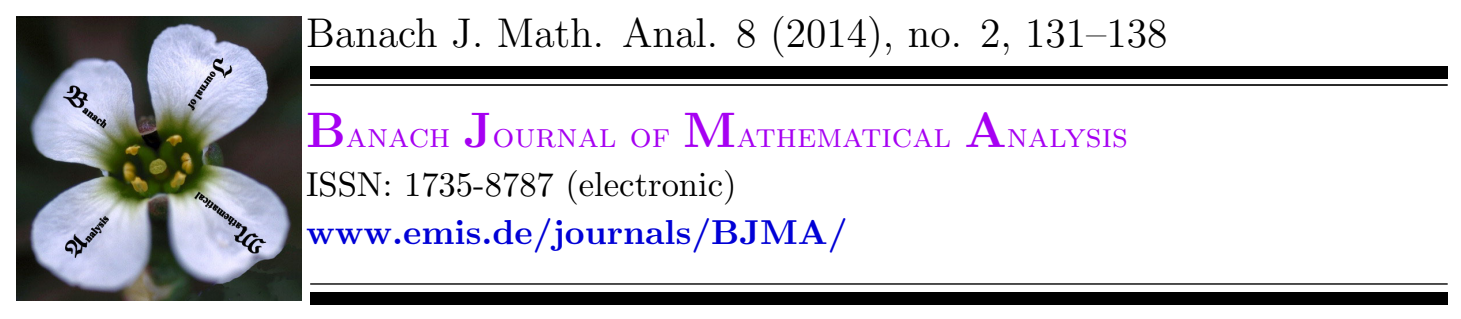

\title{
STRONG COEFFICIENT QUANTIZATION PROPERTIES IN BANACH SPACES
}

\author{
KYUGEUN CHO ${ }^{1}$, JU MYUNG KIM*2, SUN KWANG KIM ${ }^{3}$, HAN JU LEE ${ }^{4}$ \\ To the memory of Professor Edward Odell \\ Communicated by D. E. Alspach
}

\begin{abstract}
We prove that a dictionary for a Banach space $X$ has the strong coefficient quantization property if it has the same property when it restricted on the unit ball of $X$. We also obtain the same result for the strong net quantization property.
\end{abstract}

\section{INTRODUCTION AND THE MAIN RESULTS}

Dilworth, Odell, Schlumprecht and Zsák [2] introduced and investigated two natural coefficient quantization properties in Banach spaces. For $\varepsilon>0$ and $\delta>0$, a dictionary $\left(x_{i}\right)$ for a Banach space $X$, which means $X=\left[\left(x_{i}\right)\right]:=\overline{\operatorname{span}}\left(x_{i}\right)$, is said to have the $(\varepsilon, \delta)$-coefficient quantization property $((\varepsilon, \delta)$-CQP) if

$$
\mathcal{F}_{\delta}\left(\left(x_{i}\right)_{i \in F}\right):=\left\{\sum_{i \in F} n_{i} \delta x_{i}: n_{i} \in \mathbb{Z}\right\} \text { is } \varepsilon \text {-dense in }\left[\left(x_{i}\right)_{i \in F}\right]
$$

for every finite $F \subset \mathbb{N}$. We say that $\left(x_{i}\right)$ has the CQP if $\left(x_{i}\right)$ has the $(\varepsilon, \delta)$-CQP for some $\varepsilon>0$ and $\delta>0$. The following second notion is more general than the CQP. A dictionary $\left(x_{i}\right)$ is said to have the $(\varepsilon, \delta)$-net quantization property

Date: Received: Aug. 28, 2013; Accepted: Nov. 23, 2013.

* Corresponding author.

2010 Mathematics Subject Classification. Primary 46B20; Secondary 46B45.

Key words and phrases. coefficient quantizatioin property, strong coefficient quantizatioin property, net quantizatioin property, strong net quantizatioin property. 
$((\varepsilon, \delta)-\mathrm{NQP})$ if

$$
\mathcal{F}_{\delta}\left(\left(x_{i}\right)\right):=\left\{\sum_{i \in E} n_{i} \delta x_{i}: E \subset \mathbb{N} \text { finite, } n_{i} \in \mathbb{Z}\right\} \text { is } \varepsilon \text {-dense in }\left[\left(x_{i}\right)_{i \in F}\right]
$$

for every finite $F \subset \mathbb{N}$. We say that $\left(x_{i}\right)$ has the NQP if $\left(x_{i}\right)$ has the $(\varepsilon, \delta)$-NQP for some $\varepsilon>0$ and $\delta>0$. The difference of the two concepts is the support of the approximants.

One of the main results in [2] is to relax the definitions above by only requiring that one can approximate each element of the unit ball instead of the whole space. In the paper, we extend the result to strong quantizatioin versions which were also introduced in [2]. A dictionary $\left(x_{i}\right)$ is said to have the $(\varepsilon, \delta)$-strong coefficient quantization property $((\varepsilon, \delta)-\mathrm{SCQP})$ if

$$
\mathcal{F}_{\widehat{D}}\left(\left(x_{i}\right)_{i \in F}\right):=\left\{\sum_{i \in F} d_{i} x_{i}: d_{i} \in D_{i}\right\} \text { is } \varepsilon \text {-dense in }\left[\left(x_{i}\right)_{i \in F}\right]
$$

for every sequence $\widehat{D}:=\left(D_{i}\right)$ of $\delta$-nets for $\mathbb{R}$ with $0 \in D_{i}$ and for every finite $F \subset \mathbb{N}$. The $(\varepsilon, \delta)$-strong net quantization property $((\varepsilon, \delta)$-SNQP) is similarly defined by replacing the set of the approximants by

$$
\mathcal{F}_{\widehat{D}}\left(\left(x_{i}\right)\right):=\left\{\sum_{i \in E} d_{i} x_{i}: E \subset \mathbb{N} \text { finite, } d_{i} \in D_{i}\right\} .
$$

The $(\varepsilon, \delta)$-SCQP $(\operatorname{resp} .(\varepsilon, \delta)$-SNQP) implies the $(\varepsilon, 2 \delta)$-CQP (resp. $(\varepsilon, 2 \delta)$-NQP) because $2 \delta \mathbb{Z}$ is a $\delta$-net. But it is open whether or not the CQP (resp. NQP) implies the SCQP (resp. SNQP) [2, Problem 2.14(1), Question 7.1(2)].

We need the similar notations as in [2] for the SCQP and the SNQP to state our results. Suppose that $\left(x_{i}\right)$ has the SCQP (resp. SNQP). The function $\varepsilon_{s c}$ (resp. $\left.\varepsilon_{s n}\right): \mathbb{R}^{+} \rightarrow \mathbb{R}^{+}$is defined by

$$
\varepsilon_{s c}(\delta)\left(\operatorname{resp} . \varepsilon_{s n}(\delta)\right)=\inf \left\{\gamma:\left(x_{i}\right) \text { has the }(\gamma, \delta) \text {-SCQP (resp. SNQP) }\right\} .
$$

Then by the analogue for the SCQP and the SNQP of [2, Proposition 2.3(a)] the functions $\varepsilon_{s c}$ and $\varepsilon_{s n}$ are well defined.

For each $\delta>0$, we also define $\varepsilon_{s c}^{b}(\delta)$ (resp. $\left.\varepsilon_{s n}^{b}(\delta)\right)$ to be the infimum of those $\gamma>0$ such that

$$
\mathcal{F}_{\widehat{D}}\left(\left(x_{i}\right)_{i \in F}\right)\left(\operatorname{resp} . \mathcal{F}_{\widehat{D}}\left(\left(x_{i}\right)\right)\right) \text { is } \gamma \text {-dense in } \operatorname{Ball}\left(\left[\left(x_{i}\right)_{i \in F}\right]\right)
$$

for every sequence $\left(D_{i}\right)$ of $\delta$-nets for $\mathbb{R}$ with $0 \in D_{i}$ and for every finite $F \subset \mathbb{N}$.

We now have:

Theorem 1.1. Let $\left(x_{i}\right)$ be a dictionary for $X$. The following assertions are equivalent.

(a) $\left(x_{i}\right)$ has the $S C Q P$.

(b) $\varepsilon_{s c}^{b}\left(\delta_{0}\right)<1$ for some $\delta_{0}>0$.

(c) There exists a $\delta_{0}>0$ such that $\varepsilon_{s c}(\delta)=\varepsilon_{s c}^{b}(\delta)<\infty$ for all $0<\delta \leq \delta_{0}$.

Theorem 1.2. Let $\left(x_{i}\right)$ be a dictionary for $X$. The following assertions are equivalent.

(a) $\left(x_{i}\right)$ has the SNQP.

(b) $\varepsilon_{s n}^{b}\left(\delta_{0}\right)<1$ for some $\delta_{0}>0$. 
(c) There exists a $\delta_{0}>0$ such that $\varepsilon_{s n}(\delta)=\varepsilon_{s n}^{b}(\delta)<\infty$ for all $0<\delta \leq \delta_{0}$.

Theorem 1.1, which gives an affirmative answer of [2, Problem 2.14(2)], and Theorem 1.2, respectively, are strong quantization versions of [2, Theorems 2.4 and 5.3]. The basic arguments of the proofs of Theorems 1.1 and 1.2 are the same with the one of [2, Theorem 2.4]. Since the proof of Theorem 1.2 is slightly different from the one of Theorem 1.1, we only prove Theorem 1.2 among them. In view of the proof of Theorem 1.2, we remark that the $\delta_{0}>0$ in (c) of Theorem 1.2 (resp. Theorem 1.1) is the same as the $\delta_{0}>0$ in (b) of that (resp. Theorem $1.1)$.

The NQP, even the SNQP, does not imply the CQP (see [2, Theorem 5.10]). But, in [2, Theorem 5.11], it was shown that a semi-normalized basis has the CQP if and only if its every subsequence has the NQP for the closed linear span of the subsequence. We obtain the same result for the SCQP and the SNQP.

Theorem 1.3. Let $\left(x_{i}\right)$ be a semi-normalized basis for $X$. Then $\left(x_{i}\right)$ has the $S C Q P$ if and only if every subsequence $\left(x_{i_{k}}\right)$ of $\left(x_{i}\right)$ has the SNQP for $\left[\left(x_{i_{k}}\right)\right]$.

\section{Proofs of Theorems 1.2 And 1.3}

We note that $\varepsilon_{s n}^{b}: \mathbb{R}^{+} \rightarrow \mathbb{R}^{+}$is a nondecreasing function.

Proof of Theorem 1.2. (a) $\Rightarrow(\mathrm{b})$ and $(\mathrm{c}) \Rightarrow(\mathrm{a})$ are clear.

(b) $\Rightarrow(\mathrm{c})$ Choose a $t>0$ such that

$$
\varepsilon_{s n}^{b}(\delta) \leq \varepsilon_{s n}^{b}\left(\delta_{0}\right)<t<1
$$

for all $0<\delta \leq \delta_{0}$.

Now assume that $0<\delta, \bar{\delta} \leq \delta_{0}$ satisfy

$$
t \leq \frac{\delta}{\bar{\delta}} \leq \frac{1}{t}
$$

Let $\alpha>0$ be arbitrary. Let $\left(D_{i}\right)$ be a sequence of $\delta$-nets for $\mathbb{R}$ with $0 \in D_{i}$ and let $F \subset \mathbb{N}$ be finite. Let $x \in \operatorname{Ball}\left(\left[\left(x_{i}\right)_{i \in F}\right]\right)$. Then there exists a $y=$ $\sum_{i \in E} d_{i} x_{i} \in \mathcal{F}_{\widehat{D}}\left(\left(x_{i}\right)\right)$ such that $\|x-y\| \leq t$. We see that $\|(\bar{\delta} / \delta)(x-y)\| \leq 1$ and $\left(D_{i}^{\prime}\right)=\left((\bar{\delta} / \delta)\left(D_{i}-d_{i}\right)\right)$, where $d_{i}=0$ if $i \notin E$, is a sequence of $\bar{\delta}$-nets for $\mathbb{R}$ with $0 \in D_{i}^{\prime}$. Then there exists a $z=\sum_{i \in E_{0}} d_{i}^{\prime} x_{i} \in \mathcal{F}_{\widehat{D^{\prime}}}\left(\left(x_{i}\right)\right)$ such that $\|(\bar{\delta} / \delta)(x-y)-z\| \leq(1+\alpha) \varepsilon_{s n}^{b}(\bar{\delta})$. It follows that

$$
\left\|x-\left(y+\frac{\delta}{\bar{\delta}} z\right)\right\| \leq(1+\alpha) \frac{\delta}{\bar{\delta}} \varepsilon_{s n}^{b}(\bar{\delta}) .
$$

To show that $y+(\delta / \bar{\delta}) z \in \mathcal{F}_{\widehat{D}}\left(\left(x_{i}\right)\right)$, put

$$
z=\sum_{i \in E_{0} \cap E} d_{i}^{\prime} x_{i}+\sum_{i \in E_{0} \backslash E} d_{i}^{\prime} x_{i}=\sum_{i \in E_{0} \cap E} \frac{\bar{\delta}}{\delta}\left(c_{i}-d_{i}\right) x_{i}+\sum_{i \in E_{0} \backslash E} \frac{\bar{\delta}}{\delta} c_{i} x_{i},
$$


where $c_{i} \in D_{i}$. Then

$$
\begin{aligned}
& y+\frac{\delta}{\bar{\delta}} z \\
& =\sum_{i \in E} d_{i} x_{i}+\frac{\delta}{\bar{\delta}}\left(\sum_{i \in E_{0} \cap E} \frac{\bar{\delta}}{\delta}\left(c_{i}-d_{i}\right) x_{i}+\sum_{i \in E_{0} \backslash E} \frac{\bar{\delta}}{\delta} c_{i} x_{i}\right) \\
& =\sum_{i \in E \backslash E_{0}} d_{i} x_{i}+\sum_{i \in E_{0}} c_{i} x_{i} \in \mathcal{F}_{\widehat{D}}\left(\left(x_{i}\right)\right) .
\end{aligned}
$$

Thus $\varepsilon_{s n}^{b}(\delta) \leq(1+\alpha)(\delta / \bar{\delta}) \varepsilon_{s n}^{b}(\bar{\delta})$. Since $\alpha>0$ was arbitrary, we have

$$
\frac{\varepsilon_{s n}^{b}(\delta)}{\delta} \leq \frac{\varepsilon_{s n}^{b}(\bar{\delta})}{\hat{\delta}}
$$

By exchanging the roles of $\delta$ and $\bar{\delta}$ we also obtain the opposite inequality. We have shown that

$$
\frac{\varepsilon_{s n}^{b}(\delta)}{\delta}=\frac{\varepsilon_{s n}^{b}(\bar{\delta})}{\hat{\delta}}
$$

for every $0<\delta, \bar{\delta} \leq \delta_{0}$ satisfying $t \leq \delta / \bar{\delta} \leq 1 / t$. A simple verification shows that $\varepsilon_{s n}^{b}$ is linear on $\left(0, \delta_{0}\right]$.

Now, in order to complete the proof, let $\delta \in\left(0, \delta_{0}\right]$. Let $\alpha>0$ be arbitrary. Let $\left(D_{i}\right)$ be a sequence of $\delta$-nets for $\mathbb{R}$ with $0 \in D_{i}$ and let $F \subset \mathbb{N}$ be finite. Let $x \in\left[\left(x_{i}\right)_{i \in F}\right]$. If $\|x\| \geq 1$, then $\left(D_{i} /\|x\|\right)$ is a sequence of $\delta /\|x\|$-nets for $\mathbb{R}$ with $0 \in D_{i} /\|x\|$ and $\delta /\|x\| \leq \delta_{0}$. Then there exists a $\sum_{i \in E}\left(d_{i} /\|x\|\right) x_{i} \in \mathcal{F}_{\widehat{D} /\|x\|}\left(\left(x_{i}\right)\right)$ such that

$$
\left\|\frac{x}{\|x\|}-\sum_{i \in E} \frac{d_{i}}{\|x\|} x_{i}\right\| \leq(1+\alpha) \varepsilon_{s n}^{b}\left(\frac{\delta}{\|x\|}\right)=(1+\alpha) \frac{1}{\|x\|} \varepsilon_{s n}^{b}(\delta) .
$$

It follows that

$$
\left\|x-\sum_{i \in E} d_{i} x_{i}\right\| \leq(1+\alpha) \varepsilon_{s n}^{b}(\delta) .
$$

If $\|x\| \leq 1$, then clearly we can find a $(1+\alpha) \varepsilon_{s n}^{b}(\delta)$-approximant of $x$. Thus $\varepsilon_{s n}(\delta) \leq(1+\alpha) \varepsilon_{s n}^{b}(\delta)$. Since $\alpha>0$ was arbitrary, we complete the proof.

The following corollary is a strong quantization version of [2, Corollary 2.5].

Corollary 2.1. Let $\left(x_{i}\right)$ be a dictionary for $X$. Let $0<\varepsilon_{0}<1$ and $\delta_{0}>0$. If for every sequence $\left(D_{i}\right)$ of $\delta_{0}$-nets for $\mathbb{R}$ with $0 \in D_{i}$ and for every finite $F \subset \mathbb{N}$ we have

$$
\mathcal{F}_{\widehat{D}}\left(\left(x_{i}\right)\right) \text { is } \varepsilon_{0} \text {-dense in } \operatorname{Ball}\left(\left[\left(x_{i}\right)_{i \in F}\right]\right),
$$

then for all $\varepsilon>\varepsilon_{0}$,

$$
\mathcal{F}_{\widehat{D}}\left(\left(x_{i}\right)\right) \text { is } \varepsilon \text {-dense in }\left[\left(x_{i}\right)_{i \in F}\right]
$$

for every sequence $\left(D_{i}\right)$ of $\delta_{0}$-nets for $\mathbb{R}$ with $0 \in D_{i}$ and for every finite $F \subset \mathbb{N}$.

Proof. By the assumption

$$
\varepsilon_{s n}^{b}(\delta) \leq \varepsilon_{s n}^{b}\left(\delta_{0}\right) \leq \varepsilon_{0}<1
$$


for all $0<\delta \leq \delta_{0}$. We can apply the proof of Theorem 1.2 replacing $t>0$ by $\varepsilon_{0}<\varepsilon<1$ to show that $\varepsilon_{s n}(\delta) \leq \varepsilon_{s n}^{b}(\delta)$ for all $0<\delta \leq \delta_{0}$. In particular, $\varepsilon_{s n}\left(\delta_{0}\right) \leq \varepsilon_{s n}^{b}\left(\delta_{0}\right) \leq \varepsilon_{0}$. Hence we obtain the desired conclusion.

Remark 2.2. We can adapt the proof of Theorem 1.2 to show Theorem 1.1 and also obtain the analogue for Theorem 1.1 of Corollary 2.1 by replacing $\mathcal{F}_{\widehat{D}}\left(\left(x_{i}\right)\right)$ by $\mathcal{F}_{\widehat{D}}\left(\left(x_{i}\right)_{i \in F}\right)$.

Proof of Theorem 1.3. Since the SCQP is inherited by subsequences, the "only if" part is clear. In order to show the "if" part, assume that $\left(x_{i}\right)$ does not have the SCQP. We use the argument of the proof in [2, Theorem 5.11] to find a subsequence of $\left(x_{i}\right)$ failing to have the SNQP for its closed linear span.

Let $K$ be the basis constant of $\left(x_{i}\right)$ and we may assume that $\left\|x_{i}\right\| \leq 1$ for all $i$.

Step 1. For every $\delta>0$, there exists $M_{\delta} \subset \mathbb{N}$ such that $\left(x_{i}\right)_{i \in M_{\delta}}$ does not have the $(1, \delta)$-SNQP.

See the proof of Claim 1 in that of [2, Theorem 5.11] for the proof of Step 1.

Step 2. For every $n \in \mathbb{N}$, there exists a sequence $\left(D_{i, n}\right)_{i}$ of $1 / n$-nets for $\mathbb{R}$ with $0 \in D_{i}$ such that for some finite $F_{n} \subset[n+1, \infty)$ and some $y_{n} \in\left[\left(x_{i}\right)_{i \in F_{n}}\right]$, we have $\left\|y-y_{n}\right\|>2 K$ for all $y \in \mathcal{F}_{\widehat{D_{n}}}\left(\left(x_{i}\right)_{i \in F_{n}}\right)$.

Proof of Step 2. Fix $n \in \mathbb{N}$. By Step 1 there exists $M_{n} \subset \mathbb{N}$ such that $\left(x_{i}\right)_{i \in M_{n}}$ does not have the $(2 K+1,1 / n)$-SNQP. Thus there exists a sequence $\left(D_{i}\right)$ of $1 / n$ nets for $\mathbb{R}$ with $0 \in D_{i}$ such that for some finite $E_{n} \subset M_{n}$ and $z_{n}=\sum_{i \in E_{n}} a_{i} x_{i}$, we have $\left\|y-z_{n}\right\|>2 K+1$ for all $y \in \mathcal{F}_{\widehat{D}}\left(\left(x_{i}\right)_{i \in M_{n}}\right)$.

Put $w_{n}=\left.z_{n}\right|_{[1, n]}, y_{n}=\left.z_{n}\right|_{[n+1, \infty)}$, and $F_{n}=E_{n} \cap[n+1, \infty)$. For $i \in E_{n} \cap[1, n]$, choose $d_{i} \in D_{i}$ so that $\left|a_{i}-d_{i}\right| \leq 1 / n$. Let $y_{0}=\sum_{i \in[1, n] \cap E_{n}} d_{i} x_{i}$. Then $\left\|w_{n}-y_{0}\right\| \leq$ 1 , hence we have that for all $y \in \mathcal{F}_{\widehat{D}}\left(\left(x_{i}\right)_{i \in F_{n}}\right)$

$$
\begin{aligned}
& \left\|y-y_{n}\right\| \\
& =\left\|y_{0}+y-z_{n}+z_{n}-y_{n}-y_{0}\right\| \\
& \geq\left\|y_{0}+y-z_{n}\right\|-\left\|w_{n}-y_{0}\right\|>2 K+1-1=2 K .
\end{aligned}
$$

For each $k=0,1,2, \cdots$, let $\left(D_{i, n_{k}}\right)_{i}$ be the sequence of $1 / n_{k}$-nets, finite $F_{n_{k}} \subset$ $\left[n_{k}+1, \infty\right)$, and $y_{n_{k}} \in\left[\left(x_{i}\right)_{i \in F_{n_{k}}}\right]$ in Step 2, where $n_{0}=1$ and $\max F_{n_{k}}=n_{k+1}$. Put $M=\bigcup_{k \geq 0} F_{n_{k}}$. Then we have

Step 3. $\left(x_{i}\right)_{i \in M}$ does not have the SNQP.

Proof of Step 3. Suppose that $\left(x_{i}\right)_{i \in M}$ has the $(\varepsilon, \delta)$-SNQP for some $\varepsilon>0$ and $\delta>0$. Then we see that for some $\delta_{0}>0\left(x_{i}\right)_{i \in M}$ has the $(1,1 / n)$-SNQP for all $n \in \mathbb{N}$ with $1 / n<\delta_{0}$. 
Choose $k$ so that $1 / n_{k}<\delta_{0}$. Then there exists a $y \in \mathcal{F}_{\widehat{D_{n_{k}}}}\left(\left(x_{i}\right)_{i \in M}\right)$ such that $\left\|y-y_{n_{k}}\right\| \leq 1$. Thus

$$
\left\|P_{F_{n_{k}}} y-y_{n_{k}}\right\|=\left\|P_{F_{n_{k}}}\left(y-y_{n_{k}}\right)\right\| \leq 2 K
$$

where $P_{F_{n_{k}}}$ is the projection from $X$ onto $\left[\left(x_{i}\right)_{i \in F_{n_{k}}}\right]$. This is a contradiction because $P_{F_{n_{k}}} y \in \mathcal{F}_{\overline{D_{n_{k}}}}\left(\left(x_{i}\right)_{i \in F_{n_{k}}}\right)$.

\section{Bounded STRONG COEFFICIENT QUANTIZATIONS}

In [1, Definition 4.1], a bounded version, which is associated to frames for Banach spaces, of the NQP was introduced. We introduce a stronger notion of that as in the previous concept. Let $\hat{z}:=\left(z_{i}\right)$ be a sequence in a Banach space $Z$. For $\varepsilon>0, \delta>0$ and $K_{\hat{z}}>0$, a dictionary $\left(x_{i}\right)$ for $X$ is said to have the $\left(\varepsilon, \delta, K_{\hat{z}}\right)$-strong net quantization property $\left(\left(\varepsilon, \delta, K_{\hat{z}}\right)\right.$-SNQP) if for every sequence $\left(D_{i}\right)$ of $\delta$-nets for $\mathbb{R}$ with $0 \in D_{i}$, for every finite $F \subset \mathbb{N}$ and $x \in\left[\left(x_{i}\right)_{i \in F}\right]$, there exists a $\sum_{i \in E} d_{i} x_{i} \in \mathcal{F}_{\widehat{D}}\left(\left(x_{i}\right)\right)$ such that

$$
\left\|\sum_{i \in E} d_{i} z_{i}\right\|_{Z} \leq K_{\hat{z}}\|x\| \text { and }\left\|x-\sum_{i \in E} d_{i} x_{i}\right\|_{X} \leq \varepsilon .
$$

We similarly define the $\left(\varepsilon, \delta, K_{\hat{z}}\right)$ - strong coefficient quantization property $\left(\left(\varepsilon, \delta, K_{\hat{z}}\right)\right.$ $\mathrm{SCQP})$ replacing the set $\mathcal{F}_{\widehat{D}}\left(\left(x_{i}\right)\right)$ by $\mathcal{F}_{\widehat{D}}\left(\left(x_{i}\right)_{i \in F}\right)$.

We now obtain a strong quantization version of [1, Proposition 4.2].

Theorem 3.1. Let $\left(z_{i}\right)$ be a sequence in $Z$ and $\left(x_{i}\right)$ a dictionary for $X$. For $0<\varepsilon_{0}<1, \delta_{0}>0$, and $K_{0}>0$, if for every sequence $\left(D_{i}\right)$ of $\delta_{0}$-nets for $\mathbb{R}$ with $0 \in D_{i}$, for every finite $F \subset \mathbb{N}$ and $x \in \operatorname{Ball}\left(\left[\left(x_{i}\right)_{i \in F}\right]\right)$, there exists a $\sum_{i \in E} d_{i} x_{i} \in \mathcal{F}_{\widehat{D}}\left(\left(x_{i}\right)\right)$ such that

$$
\left\|\sum_{i \in E} d_{i} z_{i}\right\| \leq K_{0} \quad \text { and } \quad\left\|x-\sum_{i \in E} d_{i} x_{i}\right\| \leq \varepsilon_{0},
$$

then $\left(x_{i}\right)$ has the $\left(1, \delta_{0}, K_{\hat{z}}\right)-S N Q P$, where $K_{\hat{z}}=K_{0} \sum_{n=0}^{\infty} \varepsilon_{0}^{n}$.

Proof. This proof is very similar to the proof of [1, Proposition 4.2]. First, for every $n \in \mathbb{N}$ and $\delta \leq \varepsilon_{0}^{n-1} \delta_{0}$, we assert that for every sequence $\left(D_{i}\right)$ of $\delta$-nets for $\mathbb{R}$ with $0 \in D_{i}$, for every finite $F \subset \mathbb{N}$ and $x \in \operatorname{Ball}\left(\left[\left(x_{i}\right)_{i \in F}\right]\right)$, there exists a $\sum_{i \in E} d_{i} x_{i} \in \mathcal{F}_{\widehat{D}}\left(\left(x_{i}\right)\right)$ such that

$$
\left\|\sum_{i \in E} d_{i} z_{i}\right\| \leq K_{0} \sum_{k=0}^{n-1} \varepsilon_{0}^{k} \text { and }\left\|x-\sum_{i \in E} d_{i} x_{i}\right\| \leq \varepsilon_{0}^{n} .
$$

From our assumption, the assertion follows for the case $n=1$.

Now we assume that the assertion is true for $n \in \mathbb{N}$. Let $\delta \leq \varepsilon_{0}^{n} \delta_{0}$. Let $\left(D_{i}\right)$ be a sequence of $\delta$-nets for $\mathbb{R}$ with $0 \in D_{i}$ and let $F \subset \mathbb{N}$ be finite and $x \in \operatorname{Ball}\left(\left[\left(x_{i}\right)_{i \in F}\right]\right)$. Since $\delta \leq \varepsilon_{0}^{n-1} \delta_{0}$, we can choose $\sum_{i \in E} \tilde{d}_{i} x_{i} \in \mathcal{F}_{\widehat{D}}\left(\left(x_{i}\right)\right)$ such that

$$
\left\|\sum_{i \in E} \tilde{d}_{i} z_{i}\right\| \leq K_{0} \sum_{k=0}^{n-1} \varepsilon_{0}^{k} \text { and }\left\|x-\sum_{i \in E} \tilde{d}_{i} x_{i}\right\| \leq \varepsilon_{0}^{n} .
$$


Since $\varepsilon_{0}^{-n}\left\|x-\sum_{i \in E} \tilde{d}_{i} x_{i}\right\| \leq 1$ and $\left(\varepsilon_{0}^{-n}\left(D_{i}-\tilde{d}_{i}\right)\right)\left(\tilde{d}_{i}=0\right.$ if $\left.i \notin E\right)$ is a sequence of $\delta_{0}$-nets for $\mathbb{R}$ with $0 \in \varepsilon_{0}^{-n}\left(D_{i}-\tilde{d}_{i}\right)$, by our assumption, for some finite $E^{\prime} \subset \mathbb{N}$ we can find a $d_{i}^{\prime} \in \varepsilon_{0}^{-n}\left(D_{i}-\tilde{d}_{i}\right)$ for $i \in E^{\prime}\left(d_{i}^{\prime}=0\right.$ if $\left.i \notin E^{\prime}\right)$ such that

$$
\left\|\sum_{i \in E^{\prime}} d_{i}^{\prime} z_{i}\right\| \leq K_{0} \text { and }\left\|\varepsilon_{0}^{-n}\left(x-\sum_{i \in E} \tilde{d}_{i} x_{i}\right)-\sum_{i \in E^{\prime}} d_{i}^{\prime} x_{i}\right\| \leq \varepsilon_{0} .
$$

Hence we have that

$$
\left\|\sum_{i \in E \cup E^{\prime}}\left(\tilde{d}_{i}+\varepsilon_{0}^{n} d_{i}^{\prime}\right) z_{i}\right\| \leq K_{0} \sum_{k=0}^{n} \varepsilon_{0}^{k}
$$

and

$$
\left\|x-\sum_{i \in E \cup E^{\prime}}\left(\tilde{d}_{i}+\varepsilon_{0}^{n} d_{i}^{\prime}\right) x_{i}\right\| \leq \varepsilon_{0}^{n+1} .
$$

As in the proof of Theorem 1.2, we see that $\tilde{d}_{i}+\varepsilon_{0}^{n} d_{i}^{\prime} \in D_{i}$ for $i \in E \cup E^{\prime}$, which completes our assertion.

In order to complete the proof, let $\left(D_{i}\right)$ be a sequence of $\delta_{0}$-nets for $\mathbb{R}$ with $0 \in D_{i}$ and let $F \subset \mathbb{N}$ be finite and $x \in\left[\left(x_{i}\right)_{i \in F}\right]$.

If $\|x\| \geq 1$, then choose an $n \in \mathbb{N}$ so that $\varepsilon_{0}^{n}<1 /\|x\| \leq \varepsilon_{0}^{n-1}$. From our assertion, we can find a $\sum_{i \in E}\left(d_{i} /\|x\|\right) x_{i} \in \mathcal{F}_{\widehat{D} /\|x\|}\left(\left(x_{i}\right)\right)$ such that

$$
\left\|\sum_{i \in E} \frac{d_{i}}{\|x\|} z_{i}\right\| \leq K_{0} \sum_{k=0}^{n-1} \varepsilon_{0}^{k} \leq K_{z} \text { and }\left\|\frac{x}{\|x\|}-\sum_{i \in E} \frac{d_{i}}{\|x\|} x_{i}\right\| \leq \varepsilon_{0}^{n} .
$$

Hence

$$
\left\|\sum_{i \in E} d_{i} z_{i}\right\| \leq K_{z}\|x\| \text { and }\left\|x-\sum_{i \in E} d_{i} x_{i}\right\| \leq \varepsilon_{0}^{n}\|x\| \leq 1 .
$$

If $\|x\|<1$, then take $d_{i}=0$ for all $i \in F$.

Remark 3.2. We can also obtain the analogue for the bounded version of SCQP of Theorem 3.1 by replacing $\mathcal{F}_{\widehat{D}}\left(\left(x_{i}\right)\right)$ by $\mathcal{F}_{\widehat{D}}\left(\left(x_{i}\right)_{i \in F}\right)$.

Acknowledgement. The authors would like to thank the referee for valuable comments. The second author was supported by NRF-2013R1A1A2A10058087 funded by the Korean Government. The fourth author was supported by Basic Science Research program through the National Research Foundation of Korea (NRF) funded by the Ministry of Education, Science and Technology (NRF2012R1A1A1006869).

\section{REFERENCES}

1. P.G. Casazza, S.J. Dilworth, E. Odell, T. Schlumprecht and A. Zsák, Coefficient quantization for frames in Banach Spaces, J. Math. Anal. Appl. 348 (2008), 66-86.

2. S.J. Dilworth, E. Odell, T. Schlumprecht and A. Zsák, Coefficient Quantization in Banach Spaces, Found. Comput. Math. 8 (2008), 703-736.

${ }^{1}$ Bangmok College of Basic Studies, Myong Ji University, Yong-In, Kyung-Ki 449-728, Korea

E-mail address: kgjo@mju.ac.kr 
2 Department of Mathematical Sciences, Seoul National University, Seoul 151-747, KOREA.

E-mail address: kjm21@kaist.ac.kr

3 Department of Mathematics, Kyonggi University , Suwon 443-760, Korea.

E-mail address: sunkwang@kgu.ac.kr

4 Department of Mathematics Education, Dongguk University - Seoul, Seoul 100-715, KorEA.

E-mail address: hanjulee@dongguk.edu 